\title{
Study of gas discharge thyratron-type interrupter with sector hole in the screen in high-voltage pulse generator circuit with inductive energy storage
}

\author{
S.A. Kruglov ${ }^{1,}{ }^{*}$, N.M. Vereschagin ${ }^{1}$, A.A. Serezhin ${ }^{1}$, S.G. Shatilov ${ }^{1}$, and K.D. Agaltsov ${ }^{1}$ \\ ${ }^{1}$ Ryazan State Radio Engineering University, 59/1, Gagarina st., Ryazan, 390005, Russia
}

\begin{abstract}
The work presents experimental study results of gas discharge interrupter with a sector hole in the screen and a circular grid hole being asymmetrically placed regarding central axis as part of a high-voltage pulse generator with inductive energy storage. According to the results received the device under consideration managed to decrease minimum turn-off time by 6 times up to $30 \mathrm{~ns}$ compared to a switch with inverse hole pattern in a grid node. Limiting pulse voltage amplitude is fixed at the level of $50 \mathrm{kV}$, peak pulse power increase up to $13 \mathrm{MW}$ is achieved.
\end{abstract}

\section{Introduction}

Current stage in the development of high voltage pulse technologies can be characterised as the stage of combining modern electronic component base with new materials and traditional approaches used when developing and designing high voltage pulse generators.

According to physical principles of energy storage high voltage nanosecond pulse generators are subdivided into several groups, viz. mechanical storage practically being out of use nowadays due to their low efficiency, capacitive energy storage (CES) representing the system of capacitors connected in series or parallel with high stored specific energy of electric field and inductive energy storage (IES) usually made as solenoids with dielectric frame storing the energy of magnetic field.

To transfer energy from the storage to the load we need an additional element allowing us to switch the stored energy at a certain moment of time. Optimizing the operating mode of the storage together with the switching device is one of the tasks that can be solved with the help of high voltage pulse generators.

The operation of the generator under study is based on the method to obtain high voltage pulses caused by current cutoff by an amplitude of up to $1 \mathrm{kA}$ in a gas discharge current switch. A given method is realised in the conditions of developing nonequilibrium state of gas discharge low pressure plasma in a gap with variable cross-section (gas discharge device grid node) under the influence of electric field strength on gas discharge gap. This state causes the process of neutral atom displacement from constricted discharge

\footnotetext{
* Corresponding author: pel.rgrtu@ yandex.ru
} 
region being prevalent over the process of returning neutral gas into it. Plasma decay in the volume between grid and anode electrodes is accompanied by sharply accelerating effect of charged particles recombination. The task to develop special gas discharge current interrupters with improved discharge parameters for a high voltage pulse generator with inductive storage requires the work being performed in several stages. The first stage is the stage for design, simulation and construction of prototype current interrupters. The second stage features the research of current cut-off phenomenon in new gas discharge interrupters to determine main operating characteristics of the devices as well as the influence of grid node design on the process of current break.

A given work presents the results received from the research of thyratron type gas discharge device with sector hole in the screen and circular grid hole being asymmetrically placed regarding central axis, the comparison with the characteristics received earlier from the switch with inverse hole placement in a grid node is given.

\section{Description of a new gas discharge interrupter}

The research of gas discharge switches held before has proved the existence of direct relationship between grid node design parameters including the geometry of grid electrode holes and internal processes of low pressure plasma decay that determine the value of breaking current in a device and voltage at generator output terminal [1].

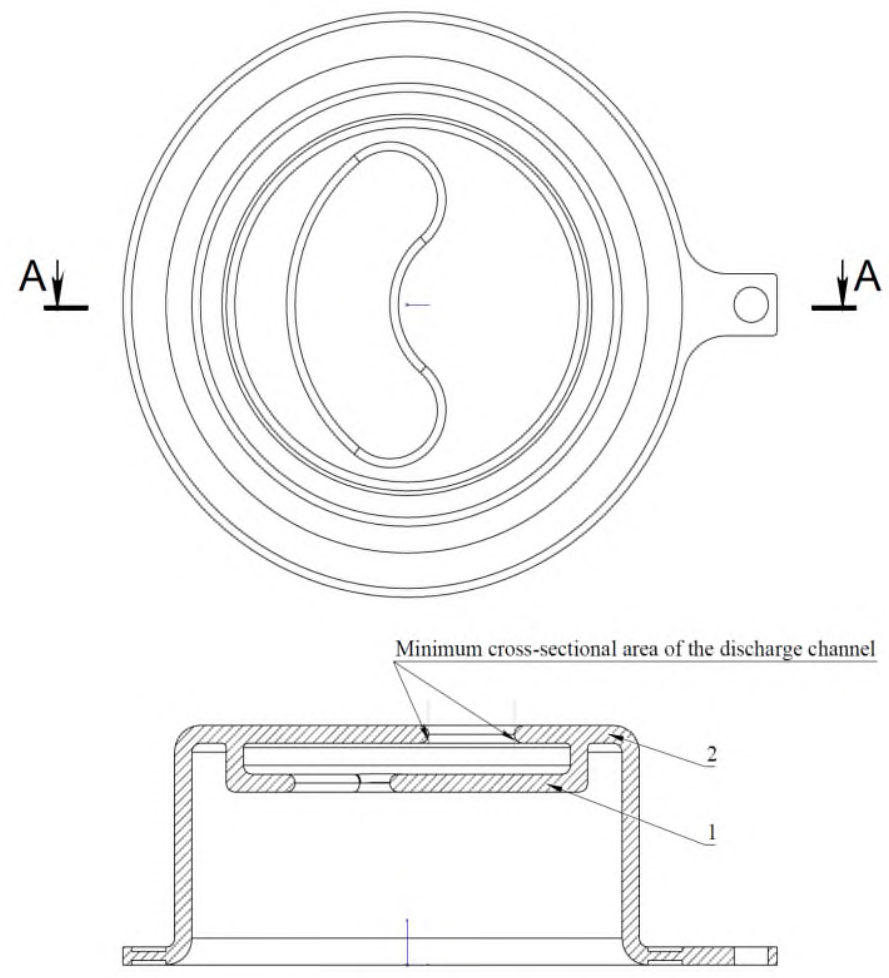

A-A $(2: 1)$

Fig. 1. Drawing of thyratron type gas discharge switch grid node with sector hole in the screen: 1 screen electrode, 2 - grid electrode. 
A drawing of grid node as well as its isometric image are given in Figures 1 and 2. As the new device has grid node design with sector hole in the screen and circular grid hole being asymmetrically placed regarding the centre a number of advantages compared to the interrupter with inverse hole placement in a grid node is provided [2]. Discharge burning process is not distributed during the time of anode current flow because of a single hole being placed both in screen and grid electrodes. Current break in this design is localised in the area of grid electrode hole (Figure 1) that helps to reduce turn-off time due to the decrease of plasma volume collected in the area of anode-grid electrodes after current selfbreak process.

Maximum electron path length from cathode region to anode one is $31,2 \mathrm{~mm}$ providing minimum ignition pressure being equal to $18 \mathrm{~Pa}$. Thicker walls and increased distance between grid node electrodes lead to lower heating [3]. Rounding hole edges allows reducing their heating and increasing threshold voltage of auto-electronic emission process under the influence of high pulse voltages. A new grid node allows us to study break phenomenon in the area of grid hole neglecting the influence of a grid screen hole. With this design a grid screen gives the possibility to maintain interrupter electrical strength at a certain degree without distorting arc formation process and interfering with the gas flowing into a grid hole area.

The results received after the simulation of electric field distribution in a new gas discharge interrupter show the depth of anode field penetration into cathode region being equal to $4.5 \mathrm{~mm}$ (Figure 3), them being correspondent with the previously received data in a device with inverse hole placement in a grid node thus having proved sufficient electric strength to be used in a high-voltage pulse generator.

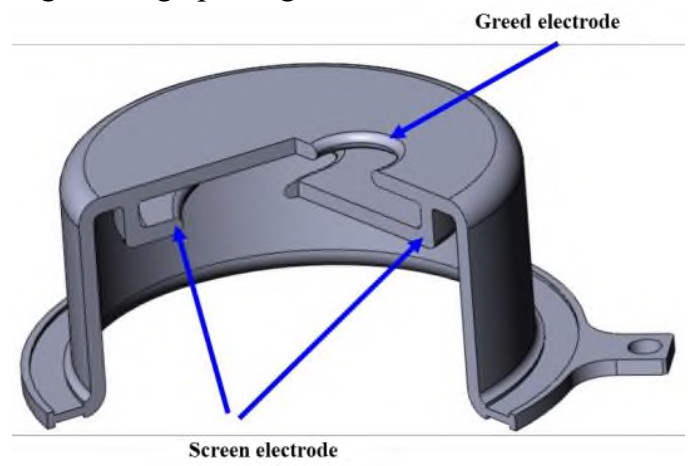

Fig. 2. Isometric image of thyratron type gas discharge switch grid node with sector hole in the screen and a circular grid hole being asymmetrically placed regarding central axis.

Anode region

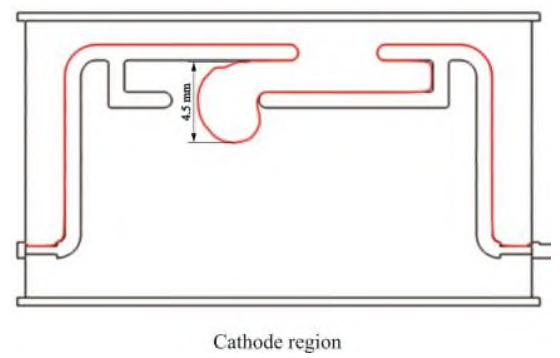

Fig. 3. Distribution of electric field with $1 \mathrm{~V}$ potential in a grid node of a new gas discharge interrupter with the voltage being $100 \mathrm{kV}$ and grid node voltage being $0 \mathrm{~V}$. 


\section{Study of a new gas discharge interrupter}

To check the performance of the device and determine its operating parameters the authors have studied the influence of pressure in the device as well as breaking current amplitude on critical charge, current break time instability (jitter), the evaluation of pressure parameters, breaking current and output pulse amplitude influence on turn off time, with limiting device parameters being also determined.

One of the parameters that determine the efficiency of current breaking process being dependent on grid node dimensions is the value of critical charge [4,5]. The pressure set in the device determines interruption moment also affecting critical charge change therefore as in the previous works the dependency of critical charge from the pressure is important to determine the range of operating pressures leading to stable current interruption. Dependences of critical charge from hydrogen pressure at different fixed power supply voltage values (Figure 4) for a new switch have shown that the range of operating pressures is within 18-23 Pa while the critical charge varies from 1 to $6 \mathrm{mC}$.

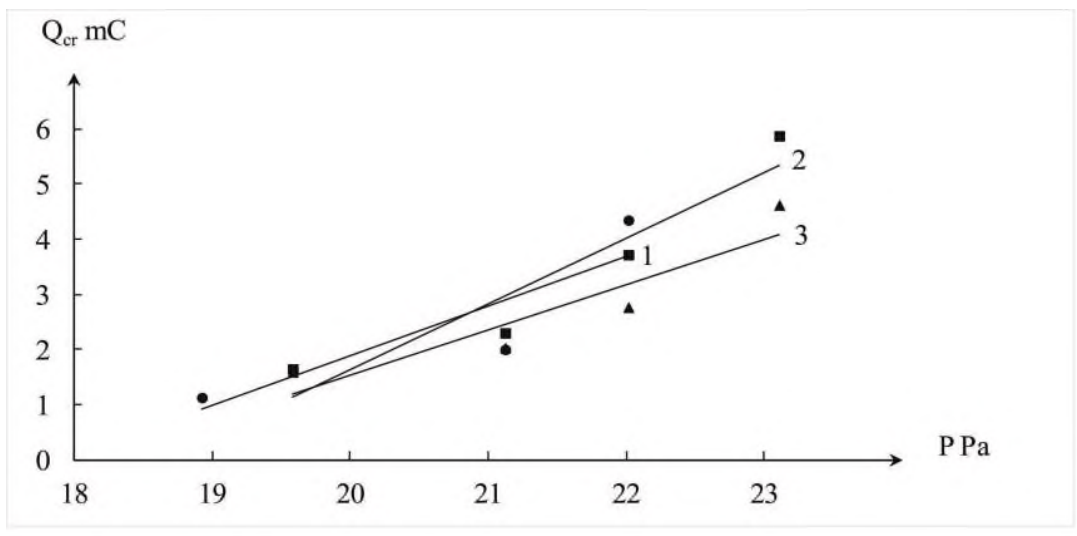

Fig. 4. Dependences of critical charge from hydrogen pressure in gas discharge switch under consideration at power supply voltages $1400 \mathrm{~V}(1), 1700 \mathrm{~V}(2), 1950 \mathrm{~V}(3), \mathrm{L}=35 \mu \mathrm{H}$, Rload $=47$ $\mathrm{Ohm}$;

Dependences show that fast growth of critical charge occurs nonlinearly together with pressure growth in the device. The range of pressures in the device under consideration is found in the region of big values compared to the switch with inverse hole placement in a grid node [6]. Unlike the switches studied before this device shows low dependency of critical charge from power supply voltage.

As we have mentioned before, the interconnection between design and energy parameters of gas discharge switch are determined by the value of breaking current flowing through the holes of a grid node as well as by critical charge connected with the area of these holes. The dependency of critical charge from breaking current amplitude (Figure 5) shows that minimum value of critical charge is located in the range of $250-350 \mathrm{~A}$, and qualitative estimate shows that the growth of breaking current value from 250 to $400 \mathrm{~A}$ (62.5\% higher) leads to 2 times charge growth (from 1.5 to $3 \mathrm{mC}$ ). Previous works [5,7-9] stated that minimum value of the charge for this parameter corresponds to maximum value of breaking current that can be achieved in a current pulse at a constant energy storage inductance value $(\mathrm{Ibr}=\operatorname{Imax})$. 


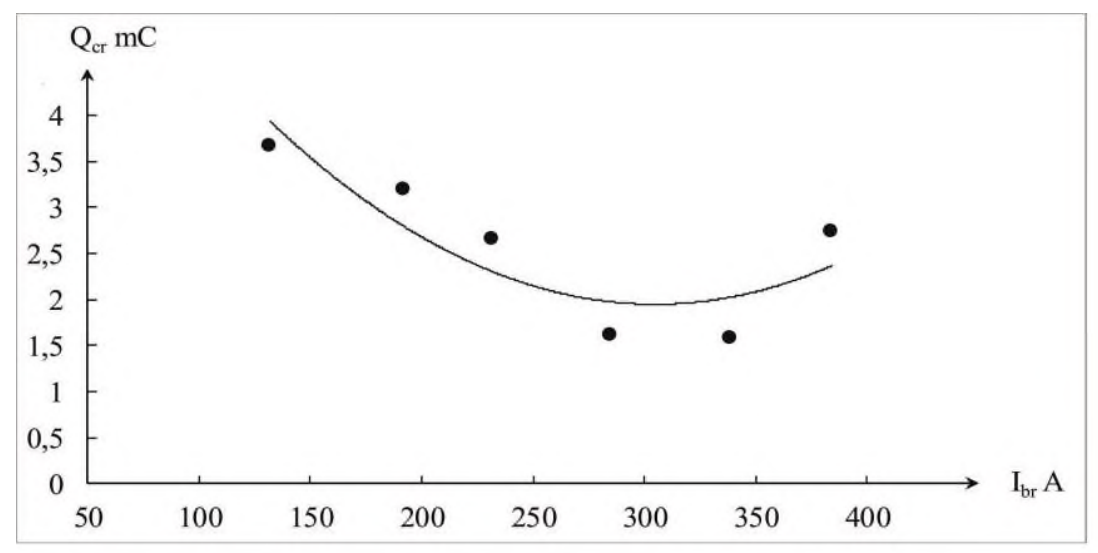

Fig. 5. Dependency of critical charge from breaking current in gas discharge switch under consideration when load pulse amplitude equals $13 \mathrm{kV}$, hydrogen pressure is $20 \mathrm{~Pa}$ and energy storage inductance is $35 \mu \mathrm{H}$.

The region on the graph adjacent to function minimum on the right side determines current break till it reaches maximum value in a pulse (leading edge of current pulse halfwave). The region on the graph of function on the left side determines current break after passing through the maximum of flowing current pulse (trailing edge of current pulse halfwave).

Similar to the earlier studies of switches with different design of grid nodes the growth of critical charge is caused by two factors: on the left side of function minimum the decrease of breaking current leads to decreasing the rate of gas displacement from a grid node, on the right side of function minimum the intensity also decreases due to the redistribution of electron speed vector. The nature of the dependence is also similar to the device under consideration $[5,7,8]$, once again confirming the assumption that maximum breaking current with constant critical charge and minimum value is certain to determine maximum current in a pulse as well as maximum efficiency of the device. For a given gas discharge switch the minimum value of critical charge at constant power supply voltage is found when working pressure is $20 \mathrm{~Pa}$.

Comparing the results received with the results of studying gas discharge switch with inverse hole placement we should note that the dependency in the range of critical charge changes for a new switch is higher than for similar dependency [6]. Increasing overlapping area between grid and screen electrodes results in the increase of initial concentration of hydrogen molecules in the volume placed between grid and screen electrodes that leads to increasing the time necessary for the gas to be displaced also increasing the number of Coulomb collisions between electrons and ions of hydrogen.

As for time parameters that characterise the operation of gas discharge switches two main parameters relating to stability and speed of switching processes have been singled out.

The first parameter is characterised as time instability of current break in a pulse (jitter). Its presence negatively influences the operation of switches as all other things being equal, jitter influences the moment when current break occurs, consequently, the moment when high voltage load pulse appears that obstructs output voltage adjustment and causes unstable generator operation. The dependency of jitter from breaking current at a fixed value of operating pressure being equal to $20 \mathrm{~Pa}$ and storage inductance of $250 \mathrm{~A}$ shows 
that the function has its minimum for breaking curent value of $250 \mathrm{~A}$ (Figure 6), jitter in this case is $300 \mathrm{~ns}$ the same as for the switch with inverse hole placement in a grid node [6].

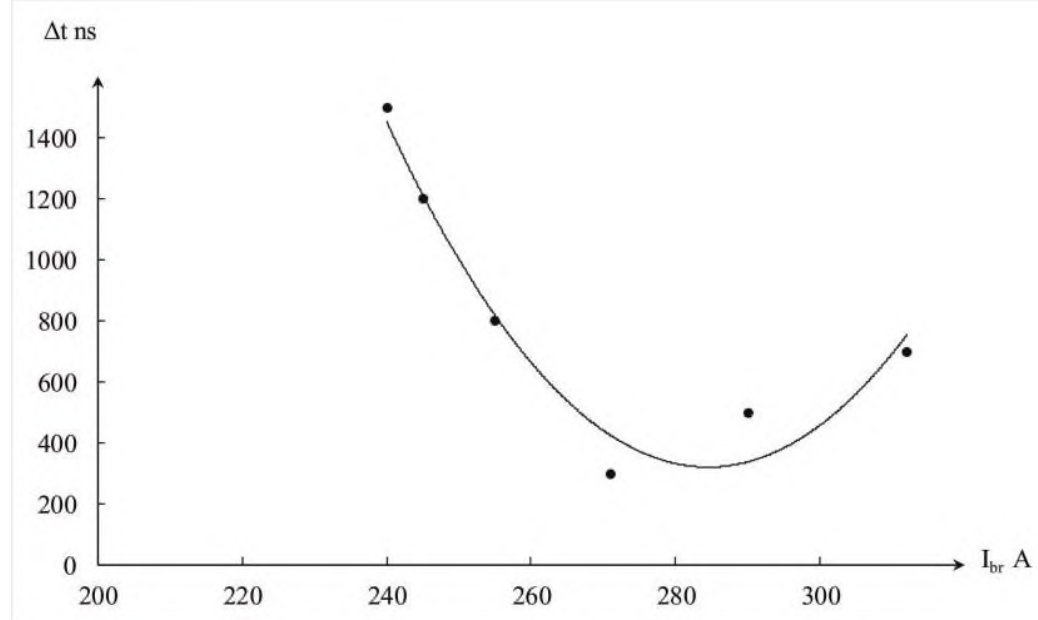

Fig. 6. Dependency of current break time instability (jitter) from break current amplitude in a new gas discharge switch when energy storage inductance equals $35 \mu \mathrm{H}$, hydrogen pressure in a device is 20 $\mathrm{Pa}$.

The second time parameter that determines the rate of broken current decay as well as the rate of increasing the leading edge of output voltage pulse in high voltage pulse generator is time of switch turn off. This parameter is characterised by the time of plasma decay placed in the volume between anode electrode and the region adjacent to a grid node hole where minimum cross-sectional area of discharge channel is found. At turn off stage a high voltage part of the generator can be represented as parallel connection of three elements: switch resistance, load resistance, energy storage inductance [10]. This peculiarity is caused by the fact that turn off time of gas discharge switch is much less than half time of circuit oscillations and time constant of capacitor recharge through the load. In this case when switching losses at turn off stage are determined using approximation of $\mathrm{x} \cdot \mathrm{ex}-1$ type, the expression for energy of losses is as follows:

$$
W_{\mathrm{sl}}=0.152 U_{\mathrm{m}} I_{\mathrm{br}} t_{\mathrm{off}}
$$

where Um - is output voltage pulse amplitude, Ibr - breaking current amplitude, toff switch turn off time [10]. Expression (1) shows that the decrease of turn off time leads to the decrease of switching losses.

Besides breaking current and output voltage the turn off time is influenced by the conditions of switch operation caused by operating pressure in a device. On the basis of received dependences of turn off time from hydrogen pressure for various fixed levels of power supply voltage (Figure 7) the following points should be mentioned: the same as in the devices already studied [5,7,8] the increase of pressure in a given gas discharge interrupter leads to the increase of turn off time, and the increase of generator power supply voltage leads to slight decrease of turn off time. Time range is 30-120 ns for pressure change from 18 to $23 \mathrm{~Pa}$. In comparison with similar dependency received for the device with inverse hole placement more than 2 times decrease of time interval is achieved [6]. 


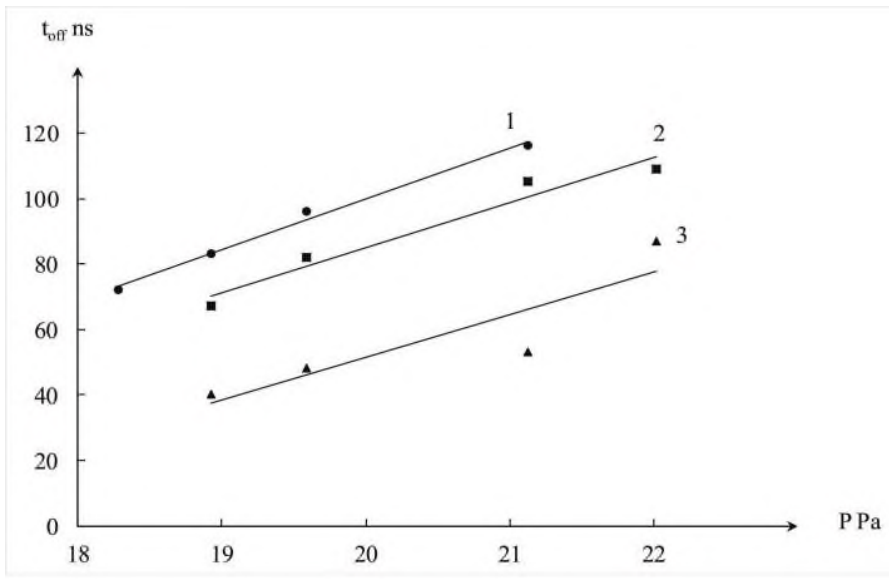

Fig. 7. Dependency of turn off time from pressure for power supply voltages $800 \mathrm{~V}$ (1), $1200 \mathrm{~V}$ (2), $1400 \mathrm{~V}$ (3) and parameters: $\mathrm{L}=35 \mu \mathrm{H}$, Rload $=47 \mathrm{Ohm}$.

The increase of power supply voltage causes the increase of breaking current which in the conditions of constant load resistance leads to the increase of output pulse voltage amplitude. As we have mentioned before, switching losses are directly proportional to the amplitude of breaking current and output voltage as well as to turn off time. In its turn, the time parameter given determines voltage slew rate in a generator output pulse according to ratio $\varepsilon=-\mathrm{Ldi} / \mathrm{dt}$, where $\mathrm{di}=\mathrm{Ibr}$ is the value of broken current, $\mathrm{dt}=$ toff - the time of switch turn off [1]. Therefore it is advisable to consider the dependences of turn off time from the amplitudes of breaking current and output pulse voltage having fixed each of these parameters.

Dependences of turn off time from breaking current show (Figure 8) that the increase of current amplitude causes the increase of turn off time that directly corresponds to the studies of similar devices made before $[5,7,8]$. It's worth mentioning that the dynamics of time range upward is slightly expressed (20\% increase) in the conditions of 2 times change of breaking current value. This slight change is caused by the increase of electron concentration in hydrogen plasma at the moment of break which results in the increase of time necessary for plasma column decay. Opposite factor that has significant impact on the change of turn off time is the amplitude output pulse voltage.

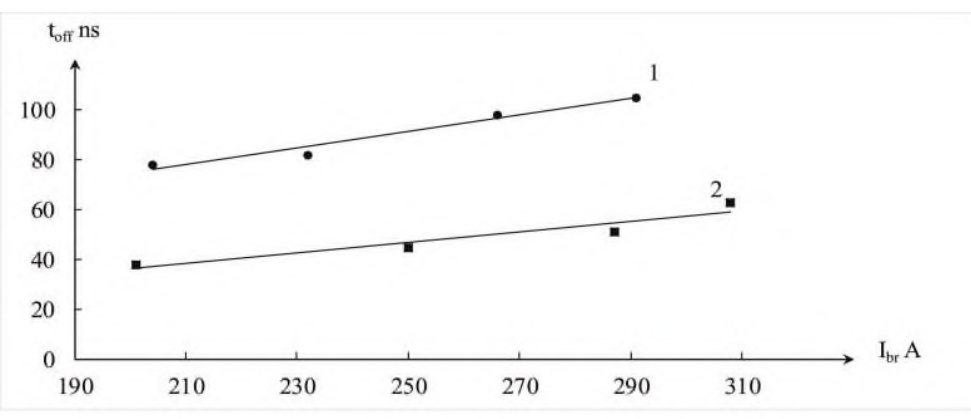

Fig. 8. Dependency of turn off time from the amplitude of breaking current for the fixed value of output pulse voltage amplitude $10 \mathrm{\kappa V}(1), 33 \mathrm{kV}(2)$, and parameters: $\mathrm{L}=35 \mu \mathrm{H}$, hydrogen pressure $20 \mathrm{~Pa}$. 
Curve number 1 in the graph is received when voltage amplitude equals $11 \mathrm{kV}$ while curve number 2 is received when voltage is $33 \mathrm{kV}$, sufficient decrease of turn off time for the whole variable range of break currents being determined.

Dependences of turn off time from output pulse voltage amplitude given in current work confirm this provision (Figure 9). Increasing voltage amplitude leads to almost 3 time decrease of turn off time value. The dependency given is explained by the fact that in the conditions of high voltage pulse formation the potential of anode electrode makes strong electric field both outside into the environment and inside the device itself as current interruption is found in anode-grid area where the distance between elctrodes is several millimeters and plasma column deionisation occurs much faster compared to the change of electron concentration inside.

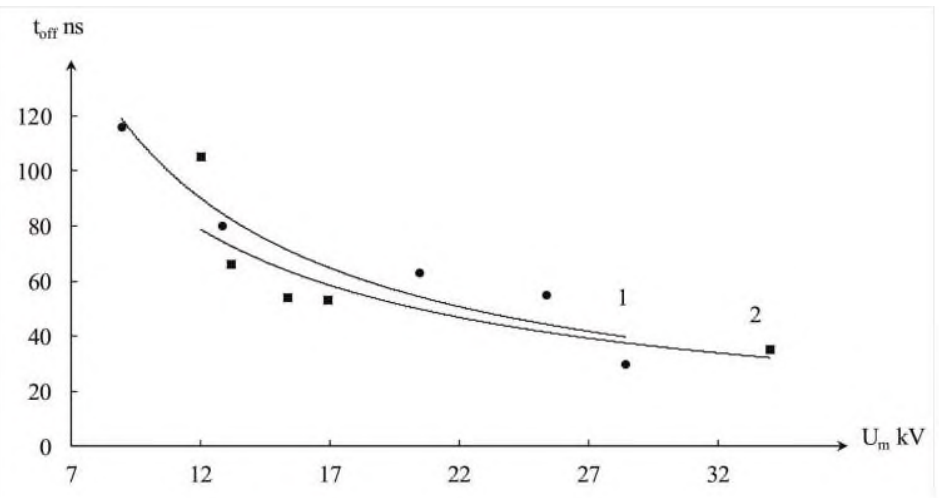

Fig. 9. Dependency of turn off time from output pulse voltage amplitude for fixed value of breaking current $200 \mathrm{~A}(1), 300 \mathrm{~A}(2)$, and parameters: $\mathrm{L}=35 \mu \mathrm{H}$, hydrogen pressure $20 \mathrm{~Pa}$.

Having made the comparison with the results received before for the interrupter with inverse hole placement in a grid node the device under consideration managed to make almost 3 times decrease of turn off time range $(30-120 \mathrm{~ns})$ as well as 6 times decrease of minimum turn off time up to $30 \mathrm{~ns}$ (Figure 10).

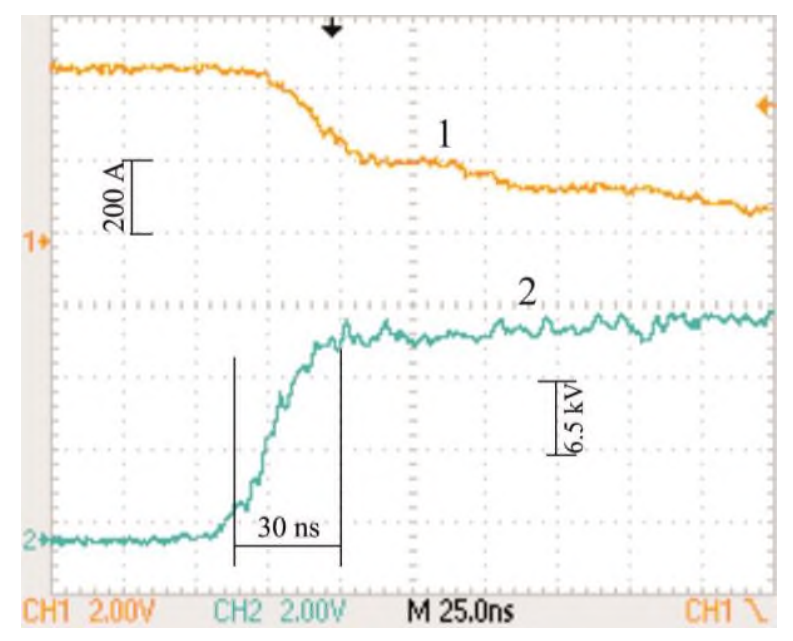

Fig. 10. Oscillograms of breaking current (1) and output voltage (2) with minimum fixed value of turn off time in a new gas discharge interrupter. 
Maximum efficiency of high voltage generator operation with inductive energy storage with gas discharge current switch is largely determined by limiting characteristics of gas discharge device. Maximum breaking current value received in a new gas discharge device is $450 \mathrm{~A}$. Such value is received with storage inductance $35 \mu \mathrm{H}$, pressure $20 \mathrm{~Pa}$, load resistance $47 \mathrm{Ohm}$, pulse amplitude in these conditions being $27 \mathrm{kV}$. Maximum voltage received is $50 \mathrm{kV}$ for the load of $1100 \mathrm{Ohm}$, breaking current $200 \mathrm{~A}$, pressure $20 \mathrm{~Pa}$, storage inductance $35 \mu \mathrm{H}$. Maximum pulse power achieved is $13 \mathrm{MW}$.

\section{Conclusion}

The work given presents the results received from the research of thyratron type gas discharge switch with sector hole in the screen and circular grid hole being asymmetrically placed regarding central axis as part of a high voltage pulse generator with inductive energy storage.

On the basis of the results received the main operating parameters of the device have been formed: operating pressure range is within $18-23 \mathrm{~Pa}$, turn off time change range is $80 \mathrm{~ns}(30-120 \mathrm{~ns})$, minimum fixed value is $30 \mathrm{~ns}$. Maximum breaking current value received in a new gas discharge device is 450 A. Maximum value of generated pulse voltage amplitude is $50 \mathrm{kV}$. Maximum power in the pulse achieved is $13 \mathrm{MW}$. Having compared the device under consideration to the device with inverse hole placement in a grid node a new switch managed to make a 6 times decrease of turn off time minimum value, $70 \%$ increase of peak pulse power, and $20 \%$ increase of limiting pulse voltage.

The work was performed as part of the state assignment of the Ministry of Science and Higher Education of the Russian Federation (FSSN-2020-0003).

\section{References}

[1] Kruglov S A 2002 Study of gas discharge current switches in the circuit with inductive energy storage: dissertation for PhD (Ryazan)

[2] Vereschagin N M, Kruglov S A, Shatilov S G, Agaltsov K D 2020 “Gas discharge current interrupter with sector hole in the screen and circular grid hole being asymmetrically placed regarding central axis” - patent for invention №2726140 (Ryazan)

[3] Rizer Yu P 1992 Physics of gas discharge: Tutorial for higher educational institutions. 2nd edition, revised and enlarged (M.: Nauka) p 536

[4] Vereschagin N M, Kruglov S A 2002 Devices and experimental technique 2

[5] Vereschagin N M, Kruglov S A, Seryozhin A A, Shatilov S G, Agaltsov K D, Pavlov M B 2017 Devices and experimental technique 6

[6] Shatilov S G 2016 Study of gas discharge current interrupters in the mode of high voltage pulse generation: dissertation for PhD (Ryazan)

[7] Kruglov S A, Karabanov S M, Vereschagin N M, Serezhin A A, Shatilov S G, Agaltsov K D, Serezhin S A, and Suvorov D V 2019 Proceedings of 2019 IEEE International Conference on Environment and Electrical Engineering and 2019 IEEE Industrial and Commercial Power Systems Europe, EEEIC/I and CPS Europe DOI: 10.1109/EEEIC.2019.8783536EID: 2-s2.0-85070830462

[8] Kruglov S A, Karabanov S M, Vereschagin N M, Serezhin A A, Shatilov S G, Agaltsov K D, and Suvorov D V 2020 Proceedings of 2020 IEEE International Conference on Environment and Electrical Engineering and 2020 IEEE Industrial and Commercial Power Systems Europe, EEEIC/I and CPS Europe 
[9] Kruglov S A, Karabanov S M, Vereschagin N M, Serezhin A A, Shatilov S G, Agaltsov K D, Zimin A I, and Suvorov D V 2018 Proceedings of 2018 IEEE International Conference on Environment and Electrical Engineering and 2018 IEEE Industrial and Commercial Power Systems Europe, EEEIC/I and CPS Europe ISBN: 978-153865185-8

[10] Vereschagin N M, Kruglov S A, Seryozhin A A, Maisuradze A I 2011 Vestnik of RSREU Ryazan 3598 